\section{$\underset{\text { hommes }}{\text { \& migrations }}$}

\section{Hommes \& migrations}

Revue française de référence sur les dynamiques

migratoires

$1315 \mid 2016$

Ondes de choc

\title{
De la délinquance à la radicalisation
}

L'exemple de Mohamed Merah

\section{Emmanuel Jovelin}

\section{(2) OpenEdition \\ 1 Journals}

\section{Édition électronique}

URL : http://journals.openedition.org/hommesmigrations/3716

DOI : 10.4000/hommesmigrations.3716

ISSN : 2262-3353

\section{Éditeur}

Musée national de l'histoire de l'immigration

\section{Édition imprimée}

Date de publication : 1 juillet 2016

Pagination : 59-68

ISBN : 978-2-919040-36-0

ISSN : 1142-852X

\section{Référence électronique}

Emmanuel Jovelin, « De la délinquance à la radicalisation », Hommes \& migrations [En ligne], 1315 |

2016, mis en ligne le 01 juillet 2019, consulté le 02 janvier 2020. URL : http://journals.openedition.org/ hommesmigrations/3716; DOI : 10.4000/hommesmigrations.3716 


\title{
DE LA DÉLINQUANCE À LA RADICALISATION L'EXEMPLE DE MOHAMED MERAH
}

Par EMMANUEL JOVELIN, professeur des universités en sociologie, chercheur au Laboratoire lorrain des sciences sociales, université de Lorraine.

\author{
Dans les différents facteurs entremêlés qui conduisent \\ les jeunes à se lancer dans le djihad, l'aspect biographique est \\ trop souvent sous-estimé. Or les passages à l'acte reposent \\ sur une généalogie directement liée au parcours de vie \\ de ceux qui les perpètrent. Dans le cas de Mohamed Merah, \\ l'embrigadement dans l'idéologie terroriste constitue \\ la dernière étape d'un long parcours d'apprentissage de \\ la violence commencé dans sa cellule familiale et poursuivi \\ dans ses rapports avec les institutions. Une trajectoire \\ accidentée qui, à travers l'accumulation des signes \\ d'une défiance profonde vis-à-vis de toute autorité, faute \\ de repères, conduit à la déviance.
}

De Khaled Kelkal en 2005 à ceux de Mohamed Merah en 2012, de Mehdi Nemmouche en 2014, des frères Kouachi et d'Amedy Coulibaly en 2015, à Mohammed Lahouaiej-Bouhlel en 2016, les auteurs des attentats récents en France et en Belgique ont des points communs : la délinquance notoire, les institutions éducatives et la prison. La question à laquelle s'attelle cet article est celle-ci : comment ces jeunes sont-ils passés de la délinquance notoire à la radicalisation ? Il s'agit de comprendre les éléments clés qui font basculer ces jeunes de l'autre côté de la barrière, en confrontant leur parcours de vie aux théories sociologiques de la déviance. Afin d'illustrer nos analyses, nous allons nous intéresser à la trajectoire de Mohamed Merah ${ }^{1}$, comme idéal type des jeunes radicalisés.

\section{De la scolarité chaotique au placement dans les institutions éducatives}

Mohamed Merah a été placé pour la première fois dans une institution éducative à l'âge de 9 ans. C'est un enfant qui a suivi une scolarité chaotique, fréquentant plusieurs établissements d'où il se fera exclure. Mohamed redouble le CP avant d'être placé à l'âge de 9 ans. À 12 ans, il est en $6^{6}$ au collège Lalande à Toulouse. Il sera absent treize demijournées entre le 17 septembre et le 8 octobre 2001. Exclu de cette école, il se fera réinscrire à Bellefontaine par l'intermédiaire des services 
sociaux. Il restera trois mois dans ce nouvel établissement avant que la principale n'alerte le procureur. Ensuite, il reprendra une scolarité en $5^{\mathrm{e}}$ au collège Anatole France. Malheureusement, dès la rentrée suivante, en octobre 2002, il agresse une fille et sera exclu pendant trois jours. Le jour de son retour, il agresse une autre jeune fille. Compte tenu de ses difficultés avérées, on lui trouvera une autre place au collège Ber-

Il s'agit de comprendre

les éléments clés qui font

basculer ces jeunes

de l'autre côté de la barrière,

en confrontant

leur parcours de vie

aux théories sociologiques de la déviance. thelot d'où il se fera renvoyer pour " coups, blessures, et menaces de mort $»^{2}$.

En $4^{e}$, il est admis au collège du Fer-à-Cheval d'où il se fera à nouveau exclure et reviendra au collège Bellefontaine pour terminer non sans mal l'année scolaire. Renvoyé de toutes les écoles qu'il a fréquentées, aucune ne voudra plus scolariser Mohamed. Les professeurs étaient dépassés par son comportement perturbateur : "Entre deux plages d'absentéisme, il se bat, hurle et s'en prend particulièrement aux filles qu'il menace et traite de sales putes ${ }^{3}$. "Son enfance se résume donc "en une interminable série d'affrontements avec sa famille, les autorités judiciaires et les différents éducateurs, tous coupables de sopposer à sa volontét ». Mohamed sera placé dans un foyer pour adolescents dangereux et en danger. D'après les services sociaux, la mère subissait également la loi de ce garçon qui la mordait et la frappait : "La mère était régulièrement maltraitée, battue à coups de manche à balai et de pierres. Kader le grand frère, qui souvent s'invite chez sa mère, donne le ton. Le jeune Merah suit les pas de son aîné5. »

L'assistante sociale adressera une lettre au procureur et le collège lancera également une alerte au procureur de la République. "La situation empire. La maman est très souvent recueillie par une voisine. Mohamed passe chez lui tous les matins, il est le seul à pouvoir rentrer dans l'appartement quand son frère $y$ est. Et il est totalement soumis à ce frère. Il avoue bien volontiers que c'est son frère qui lui donne tous les mauvais conseils, de comportement, de révolte, mais ne peut s'empêcher de suivre et d'imiter Kader ${ }^{6}$. "

Merah grandit dans un environnement où il est exposé à des comportements défavorables au respect de la loi. Les mécanismes de l'apprentissage de la délinquance ont été transmis dans la famille, son frère étant un modèle pour lui. Suivant Gabriel Tarde, "le criminel imite toujours quelqu'un, même lorsqu'il invente, c'est-à-dire lorsqu'il combine utilement des imitations de sources diverses ${ }^{7}$ ».

La principale du collège Bellefontaine saisit le juge des enfants le 18 janvier 2002. Le même jour, ce dernier ordonne le placement immédiat de Mohamed Merah dans un foyer à Saint-Gaudens pour une durée de 12 mois. Lors de sa présentation à l'audience devant le juge des enfants, le 24 janvier, en vue de décider des retours en week-end, Mohamed s'enfuit, parce que sa mère ne souhaitait pas le recevoir chez elle. Sa sœur ainsi que l'assistante sociale tenteront de le raisonner mais il ne voulait rien entendre. Il suivra de loin les deux femmes "en les injuriant copieusement, renverse des poubelles sur les capots des voitures en stationnement, tord les essuie-glaces des véhicules et assène quelques coups de pied dans les portières. Madame Augier, l'assistante sociale, essaie de le maîtriser et de le calmer, celui-ci se retourne et décroche un violent coup de poing à la figure de l'assistante sociale. Lèvres ouvertes, cette dernière doit être hospitalisée ${ }^{8}{ }$.

\section{Une socialisation à la délinquance}

Ce jeune finira par réintégrer le foyer de SaintGaudens où les actes délictueux seront nombreux, comme le montre cet extrait de rapport : «Il multiplie les incidents et les agressions, systématiquement sur les adolescentes, qui nous demandent de les protéger et de fermer leur chambre à clé. Chaque jour, nous devons intervenir pour une dégradation, un vol, un conflit, une agression dont Mohamed est l'auteur. (...) Mohamed a une attitude exécrable au foyer. Il injurie, 
insulte les filles, ne supporte pas l'autorité des adultes, ni les frustrations. (...) Il serait nécessaire d'attacher un adulte à sa surveillance exclusive, tant à l'intérieur qu’à l'extérieur de l'établissement ${ }^{9}$. "

Le 5 février, Mohamed Merah est convoqué devant le juge des enfants. Il finira une fois de plus par fuguer. Retrouvé par le directeur du Centre d'action éducative, "il exprime sa souffrance et admet que ce serait utile pour lui de regagner le foyer ». Mais, en route, il fugue une deuxième fois et finit par regagner le foyer vers 23 heures Quelques jours plus tard, Mohamed part en séjour de ski avec ses camarades de foyer. Les problèmes persistent. Il monte sur le toit du chalet en balançant des projectiles. L'intervention de trois éducateurs sera nécessaire pour le faire redescendre. Le 12 février 2002, le foyer mettra fin au placement du jeune Merah.

Mohamed, qui violentait les autres, était aussi un garçon maltraité. Il a subi des violences de la part de son frère, qui l'avait attaché à un lit à 2 heures du matin comme le montre un rapport de l'hôpital : Mohamed «a été victime de multiples violences de caractères divers qui auraient été infligées dans un contexte familial (...) au domicile : attaché par les poignets et les chevilles, allongé sur un lit, coiffé d'un casque de moto et frappé par coups de poing, coups de balai, contraint à manger de la nourriture avariée et, enfin, abandonné seul, ligoté. Il se plaint depuis lors de céphalées ${ }^{10} "$.

En 2003, il y aura de nouvelles violences et de nouveaux placements : "Le comportement de Mohamed n'évolue pas. Les éducateurs finissent à l'hôpital, le matériel est dégradé, les insultes à caractère sexuel sont de plus en plus précises et les agressions sur ses camarades, surtout les filles font partie de la norme de l'ado Merah ${ }^{11}$. " On peut lire ainsi dans le rapport de l'éducatrice du foyer de transition où il avait été placé après avoir été expulsé en 2002 : "Il est très repérable que la violence est un procédé habituel pour Mohamed. Il a une importante intolérance à la frustration (...), il distribue de virulentes insultes à caractère raciste. Il a insulté, tapé, craché sur un éducateur qui souhaitait qu'il éteigne la télévision. Il n'a aucune limite lorsqu'il démarre dans la violence ${ }^{12}$. "

Au-delà de la violence de Mohamed, Abdelkader, son frère, arrange un mariage pour sa mère avec le père de Sabri Essid, grande figure du salafisme toulousain. Le marié se fait virer de l'appartement par Mohamed qui le frappe. Âgé de 16 ans et demi en 2005, il sera placé dans un foyer d'urgence où les incidents se multiplient : «Le 6 janvier, altercation avec le veilleur de nuit et vitres brisées de la salle à manger. (...) le 10 janvier, il insulte une hébergée au Centre en lui faisant des remarques désobligeantes et se bagarre avec la jeune fille. Mohamed fugue et reviendra plus tard. Le 13 janvier, il décide d'aller au cinéma, refus des éducateurs, il casse tout ce qu'il trouve sur son passage. Les éducateurs cèdent devant la violence de l'adolescent; le 18 janvier, refus de se coucher, disputes avec les éducateurs, le 19 janvier le foyer demande la fin du placement ${ }^{13}$."

L'incident est raconté ainsi par la directrice : « Il est dans la provocation et cherche visiblement l'affrontement masMerah grandit dans un environnement où il est exposé à des comportements défavorables au respect de la loi. Les mécanismes de l'apprentissage de la délinquance ont été transmis dans la famille, son frère étant un modèle pour lui. culin. Plusieurs fois, l'éducateur tente de mettre un terme à l'altercation, mais Mohamed revient à la charge. Mohamed s'acharne à coup de pied et de poings. Assommé par un violent coup de poing au visage, l'éducateur se retire alors que d'autres membres du personnel barrent le passage. Mohamed ne décolère pas, (...) il continue à insulter le personnel. Nous lui ouvrons la porte et il part chez sa soeur. Nous avons contacté les services de police, lesquels sont arrivés après le départ de Mohamed. Ils ont entendu l'éducateur agressé et sont allés interpeller Mohamed au domicile de sa sœur. L'éducateur ayant reçu plusieurs coups violents au visage s'est rendu à l'hôpital ${ }^{14}$. " Mohamed sera " encore » exclu du foyer et se donnera de plus en plus une identité de délinquant, qui lui collera définitivement à la peau. 


\section{La défaillance institutionnelle}

La trajectoire de Merah ne diffère pas sensiblement des délinquants classiques que nous avons vus passer dans les institutions éducatives. Routier institutionnel, il connaissait les ficelles des institutions éducatives et savait en jouer. Reste à savoir comment il en est arrivé là ? Tous les éducateurs vous diront qu'il s'agit du manque d'une autorité régulatrice et d'un système institutionnel incapable de contenir un

La trajectoire de Merah ne diffère pas sensiblement des délinquants classiques que nous avons vus passer dans les institutions

éducatives. Routier institutionnel, il connaissait les ficelles des institutions éducatives et savait en jouer. jeune en danger et dangereux pour lui-même. Au lieu de développer la pédagogie du déplacement, ce jeune continuera à être placé à Toulouse non loin de son environnement classique, ce qui constitue une erreur grave. Mais la faute incombe-t-elle aux travailleurs sociaux, qui ne sont que les exécutants des décisions judiciaires ? À notre sens, la construction de cette personnalité délinquante qui s'est sentie toute puissante incomberait, entre autres, au système de la protection judiciaire de la jeunesse qui n'a pratiquement jamais innové en matière de prise en charge, par manque de moyens suffisants. Voilà, en 2002, comment Cécile Metenier, psychologue expert du tribunal de Toulouse, décrit Mohamed Merah dans un rapport destiné au juge des enfants, suite à l'une de ses innombrables agressions. Il n'a alors que 14 ans : «L'autre est perçu comme un danger potentiel et met Mohamed Merah en position de se méfier ou de se défendre d'un préjudice souvent imaginaire. (...) Il n'envisage pas les répercussions de ses actes sur les personnes qu'il agresse, puisqu'il se place toujours lui-même en position de victime ${ }^{15}$. "Voilà un jeune homme qui a un long apprentissage de la violence et de la haine. La réponse du juge des enfants sera le placement dans une autre institution, au lieu de mobiliser des moyens pour une pédagogie de déplacement face à ces types de jeunes qui nécessitent un encadrement individuel dans des chantiers humanitaires (par exemple), dont on connaît les résultats positifs.

\section{La défaillance de l'autorité parentale}

Mohamed est un garçon qui a grandi dans la toute-puissance, jusqu' ${ }^{16}$, les discours des parents, comme ceux de la mère de Merah qui est allée se plaindre plusieurs fois à l"enracinement historique des mutations actuelles de la famille, comme l'a souligné Irène Théry ${ }^{17}$ : "C'est parce que la mutation est structurelle mais inachevée et inassumée, que surgissent les principales difficultés au sein des familles ${ }^{18}$." Pour l'auteure, le nouveau pacte de filiation demeure incertain. Les places des uns et des autres semblent brouillées. Les relations entre parents et enfants " moins rigides, moins distantes et moins autoritaires » se traduisent par le souci de respecter l'enfant dès le plus jeune âge, ce dernier devenant un partenaire. Mais c'est justement ce qui est dénoncé par les parents que nous avons interviewés: "On n'a plus le droit de rien faire, ils ont tout aujourd'hui ${ }^{19}$." Des parents ont parlé d'une'une prise de pouvoir des enfants sur eux, s'arrogeant parfois la place du père. Tout cela montre qu'il n'est pas facile " d'assurer à l'enfant son développement propre, en relation avec la vie qui est née avec lui... et de lui enseigner un monde qui a commencé avant sa naissance, qui continuera après sa mort et dans lequel il doit passer sa $v i e^{20}$. L'éducation n'est pas quelque chose de facile,

15. Ibid., pp. 15-16. 16. II s'agit d'une recherche menée auprès des jeunes qui violentent leurs parents et auprès de ces derniers dans le Nord de la France. Voir pour cette recherche, Emmanuel Jovelin, «Le sociologue, l'éducateur, et les jeunes placés dans les institutions éducatives ", in Emmanuel Jovelin (dir.), Immigration, déviance et travail social. De la sociologie interactionniste à la sociologie de la reconnaissance, HDR, université de Lille 1, 2010. 17. Irène Théry, Couple, filiation et parenté aujourd'hui. Le droit face aux mutations de la famille et de la vie privée, Paris, Odile Jacob, 1998. 18. Ibid., p. 67. 19. Emmanuel Jovelin, "Le sociologue, l'éducateur, et les jeunes placés dans les institutions éducatives ", op. cit. 20. Hannah Arendt, La crise de la culture, Paris, Gallimard, 1991, cité par Irène Théry, op. cit., p. 74. 
semblent nous dire les mères de Merah, de Coulibaly, de Nemmouche, de Kouachi, etc. Leur désarroi révèle les difficultés de l'exercice de la fonction parentale.

Comment Merah pouvait-il se construire une identité positive "lorsque l'on vit dans un foyer éclaté, un père absent, une mère démissionnaire, un grand frère qui sème la terreur et parle avec ses poings, un oncle qui ne rate pas une occasion pour insulter les juifs, lorsquion est rejeté par les siens ${ }^{21}$ »? Se pose ici la question du lien social, comme l'a soulignée Émile Durkheim : l'intégration d'un individu dans un groupe repose sur le fait d'être "incorporé à une unité sociale quelconque et d'en faire véritablement partie... Pour qu'un homme soit un être moral, il faut qu'il tienne à autre chose qu'à lui-même. Il faut qu'il se sente solidaire à une société si humble soit-elle ${ }^{22}$ ». À quoi Mohamed Merah tenait-il ? À sa sœur? À son frère ? À ses copains ? À sa mère ? Il fallait encore que Mohamed Merah sente qu'il faisait partie d'un groupe social réprouvant les actes délictueux. Or cela n'était pas le cas. Chez Merah, le passage à l'acte était inévitable parce que «le lien à la société s'était affaibli voire rompu, rendant ainsi inopérante l'action régulatrice du groupe social », comme l'avait déjà suggéré Émile Durkheim.

\section{Un scénario de la violence}

Dans notre recherche évoquée ci-dessus ${ }^{23}$, les manifestations de violence expliquées par les parents étaient relativement similaires, suivant un " crescendo » dans les actes et les attitudes. Tous les parents parlent du ton qui monte, du rapport de force entre eux et leurs enfants, suivis des menaces faites à leur encontre. Tous les parents se disent victimes d'insultes de la part de leurs enfants qui crachent sur eux, les bousculent, les frappent (comme Merah), allant même pour un cas jusqu'à leur casser des doigts, à bloquer les cervicales. Ce qui nous a marqué dans cette phase de violence (de même par rapport à Merah), c'est l'existence systématique d'un scénario, d'un enchaînement de comportements, d'actes qui conduisent des adolescents à des comportements violents à l'égard de leurs parents, de leurs camarades, de leurs éducateurs, comme nous a expliqué Madame Lambert à propos de sa fille : "Si je la prends, la lève de sa chaise et lui dis tu fais la vaisselle ou À quoi Mohamed Merah tenait-il ? À sa sœur? À son frère ? À ses copains ? À sa mère? Il fallait encore que Mohamed Merah sente qu'il faisait partie d'un groupe social réprouvant les actes délictueux.

Or cela n'était pas le cas. alors tu seras punie de ça ou de ça, elle commence ta gueule, tu m'emmerdes... tu me fais chier... puis... tais-toi sale vieille, tu ne peux pas marcher avec tes jambes... elle tape dans les portes, elle dit tu me fais chier, elle m'insulte de grosse puis... elle explose... beaucoup d'engueulades, des portes qui claquent, des violences verbales... puis elle s'enfermait...ça a commencé verbalement puis ça a été physiquement... elle avait levé la main, elle m’a poussé... elle m'a craché dessus ${ }^{24} \ldots$ »

Ce discours montre le besoin de l'adolescent de réfuter le cadre posé par les parents. Cette attitude ne diffère pas de celle du jeune Merah, futur djihadiste. On voit ici la complexité du phénomène de violence qui conjugue des états physiologiques non régulés, exacerbés, propres à l'adolescent avec le besoin de réfuter les cadres parentaux et sociétaux pour se construire. On peut également questionner, dans ce genre de relations difficiles, le juste équilibre, la bonne distance à établir entre l'adulte et l'enfant sans pour autant " démissionner » dans sa fonction parentale.

Ici prennent aussi sens les questions relatives à la non-intégration des règles soulevées par de nombreux auteurs. Les parents qui ont du succès sont ceux capables d'utiliser des stratégies, de trouver des solutions aux problèmes. Pour cela, il faut reconnaitre les situations qui risquent de dégénérer 
et intervenir tôt. Les parents de notre échantillon " se taisaient » comme la mère de Merah, au lieu de court-circuiter le processus pouvant amener à l'affrontement. Les parents de

On voit ici la complexité du phénomène de violence

qui conjugue des états physiologiques non régulés,

exacerbés, propres à l'adolescent avec le besoin de réfuter les cadres parentaux et sociétaux pour se construire. Merah avaient des difficultés à proposer des solutions faisant en sorte que l'adolescent ne perde pas la face. Comme le souligne Marc Ouimet ${ }^{25}$, l'utilisation d'une stratégie autoritaire pose des problèmes parce que ces enfants, comme Merah, ont appris que la coercition et la manipulation sont des moyens intéressants pour arriver à leurs fins. L'ouvrage de Sheldon et Eleanor Gluek, publié en $1950^{26}$, nous a appris que les enfants mal suivis par leurs parents, qui vivent dans des familles sans cohésion, auraient plus de chances de devenir des délinquants persistants.

Quant au style éducatif, nous avons remarqué au cours des entretiens et des visites dans les familles ${ }^{27}$ que, dans la majorité des familles, se dessinait une tendance plutôt « traditionnelle » fondée sur le respect des adultes, l'autorité et la division des rôles et des sexes. Peu de familles se distinguaient nettement en usant du modèle horizontal favorisant l'autonomie, le dialogue et la négociation. Cependant, pour un certain nombre d'entre elles, les parents naviguaient entre deux modèles: l'autoritarisme et la permissivité. Le cas Merah est un exemple concret de cette pratique. Or se situer entre ces deux modèles est très déstructurant pour les enfants qui, par moments, ne savent pas quelle direction prendre. Dans ces familles désorientées, on se singularise par un délabrement éducatif : perte de vigilance des parents à l'égard des enfants défaillants, une autorité relâchée, inconstante, tantôt sévère, tantôt clémente, des enfants rejetés par les parents, et des enfants faiblement attachés à ces derniers. Merah a grandi dans une famille où l'on pourrait parler de vide éducatif, de non-éduca- tion résultant de graves perturbations familiales ${ }^{28}$. Concernant Merah, comme pour les autres délinquants, on peut se référer à Durkheim qui met la question de l'autorité au centre de sa réflexion et en fait un leitmotiv. Il précise qu'il ne saurait véritablement y avoir de société, ni de moralité en l'absence "d'une autorité s'exerçant sur la pensée et le comportement des individus ${ }^{29}$. » Auprès des parents violentés par leurs enfants, nous avons croisé des problèmes "classiques » qu'on rencontre dans certaines familles : injustice, jalousie, heurts à l'adolescence, disputes... mais la différence se trouve dans l'exacerbation des expressions violentes entre enfants et parents. Ces violences, avons-nous vu, renvoient, entre autres, à la question de l'autorité (parents/enfants), de la tolérance à la différence (entre enfants), au manque de respect, mais aussi au contexte violent dans lequel baignent les protagonistes. Le fait que les parents se sentent niés dans leur autorité laisse présager qu'il existe un écart entre leur conception de l'autorité et la manière dont leurs enfants l'appréhendent et la contestent. Nous avons vu que l'autorité des parents est mise à mal, non seulement par la difficulté des enfants à intégrer les principes qu'ils posent, mais également par la décrédibilisation des figures d'autorité et notamment celles de leurs parents qui se sentent "destitués » dans leurs fonctions parentales. Alors que les parents soulignent les difficultés à se faire respecter par leurs enfants, ces derniers en font de même, en soulignant de leur côté un cadre peu adapté à leurs attentes, besoins et exigences qui divergent de celles de leurs parents.

\section{L'amplification de la délinquance ou de l'impunité à la toute puissance}

On peut facilement appliquer ici la théorie de l'étiquetage qui, au départ, est l'œuvre de Frank Tannenbaum, avant qu'Edwin Lemert et Howard 
Becker ne lui donnent ses lettres de noblesse. Dès 1938, Tannenbaum a évoqué le rôle de la communauté dans le déclenchement de la carrière délinquante. Tout commence par l'accumulation de petits méfaits, qui vont de plus en plus s'amplifier et conduiront au ras-le-bol de la communauté. L'enfant sera progressivement étiqueté et vivra avec dans un autre monde, celui de la délinquance. Dans ses travaux, Tannenbaum décrit « un processus de fabrication de délinquant par ségrégation, étiquetage et accentuation ${ }^{30}$ ». À sa suite, Lemert propose une théorie du comportement déviant basée sur la réaction sociale. Tout d'abord, c'est la réaction sociale diffuse et ensuite institutionnalisée, comme nous l'avons vu par rapport à Merah, «qui transforme le comportement déviant en un fait central de la trajectoire de la personne et contribue à le renforcer $^{31}$ ». Donc, pour Lemert, ce n'est pas le comportement déviant qui conduit au contrôle social mais bien l'inverse, parce que pour lui le phénomène fondateur de la déviance serait constitué par "les réactions de la société qui tendent à désapprouver, dégrader et isoler l'individu ${ }^{32} »$. Becker a complété les thèses de Lemert et de Tannenbaum en précisant que la déviance n'est pas une propriété du comportement lui-même, mais de l'interaction entre la personne qui commet l'acte et les institutions chargées de réagir à cet acte. Dès lors, «ce ne sont pas les motifs qui créent le comportement déviant mais justement l'inverse. C'est la pratique déviante qui peu à peu construit au fil du temps la motivation déviante ${ }^{33}$ ».

Le processus de l'amplification de la délinquance qui conduira Merah en prison va se construire ainsi : il participe à un vol de cartons de parfum dans un camion que lui et ses complices prendront le soin d'incendier. Cette affaire attirera l'attention de la police : "L'exploitation des traces papillaires relevées sur les emballages de parfums conduisait à l'identification de quatre personnes. Il s'agissait d'un majeur Mercadal Frédéric et de trois mineurs,
Merah Mohamed, Djourdem Toufik et Keller Mike, tous connus défavorablement de nos services ${ }^{34}$. " Les quatre jeunes vont nier les faits et seront relaxés. Mohamed échappe encore une fois à la justice. Il vole une BMW X5, une course-poursuite s'engagera avec la police, mais la police ne parviendra pas à l'identifier. Il avouera ces faits lors de la conversation avec la police durant le siège de son appartement. En 2006, lui et son copain frappent un jeune de 18 ans dans la rue pour lui voler son portable. Mohamed frappe également son oncle qui tente de le raisonner. Avec un ami, ils agressent un jeune et lui volent sa moto, une

Honda 250. Il se fera repé- Le processus de l'amplification rer et interpeller par la de la délinquance qui police. En 2006, il est fiché conduira Merah en prison va « $\mathrm{S}$ » (risque d'atteinte à la se construire ainsi : il participe sûreté de l'État) «en raison à un vol de cartons de parfum de ses nombreuses relations dans un camion que lui avec les milieux salafistes ». et ses complices prendront Nous pouvons reprendre la le soin d'incendier. question d'Howard Becker à propos de la récidive : «Pourquoi certains individus continuent de passer à l'acte et nous, qui pensons être normaux, ne passons pas à l'acte? " Howard Becker explique que nous, qui croyons être normaux, avons des conventions qui nous lient aux autres. Nous avons donc tout à perdre, contrairement aux multirécidivistes. Les travailleurs sociaux ont constaté que, chez Merah, il n'y avait pas d'intégration de valeurs morales comme le respect, par exemple. La réputation de «bad boy » qu'il s'est (qu'on lui a) construit était plus importante pour lui.

Devant un passage à l'acte délinquant, un adolescent peut décider d'agir ou de s'abstenir. La morale peut être une variable le dissuadant d'agir. Le jugement moral est ici considéré dans sa relation entre une situation donnée, entre les notions de bien et de mal que le sujet aura intériorisées et qui débouchent sur l'évaluation de la situation et donc sur l'éventualité 
de l'action. Cependant, on rencontre souvent chez beaucoup d'adolescents une confusion entre ce qui est bien et ce qui est permis, voire ce qui échappe aux sanctions, et entre

De l'identité héritée (valeurs familiales que les djihadistes ont

des difficultés à faire leurs)

à l'identité rêvée (travail, famille,

maison), et enfin à l'identité

construite (délinquance,

radicalisation, terrorisme), l'islam

pourrait être considéré comme

une opportunité de rencontre

qui permet à certains jeunes de

se donner " une licence » pour agir,

de s'octroyer un pouvoir afin

de transgresser tous les interdits

des sociétés occidentales.

ce qui est mal et ce qui est interdit ${ }^{35}$. Les parents que nous avons rencontrés se posaient des questions sur l'intégration de la « morale », pensant que cela se fait normalement. Selon les parents découragés, l'imposition des règles n'était plus de mise parce que, de toute façon, leurs enfants " n’obéissaient pas ", ce qui les conduit à la démission, installe les adolescents dans la toute-puissance et suscite des actes de violences caractérisées. Cependant, cela ne signifie pas, comme le suggérait David Matza ${ }^{36}$, que les délinquants sont moralement coupés de la société environnante. Leur expérience est celle « d'une oscillation constante entre deux mondes de valeurs qui se confondent. Le monde de groupe de pairs et celui des parents. Ce doute met le jeune dans une situation statutaire qui le pousse à alterner ses engagements (être un enfant sage ou un délinquant?) $)^{37}$ ".

\section{De la prison à l'islamisme : le processus de radicalisation}

Mohamed Merah atteint la majorité et rien ne semble l'arrêter : il arrache le sac à main d'une femme, la menace d'un couteau. Il est interpellé. Comme il est déjà condamné à plusieurs reprises avec sursis pour " un total de 9 mois et une dizaine d'avertissements $^{38}$ " lorsqu'il était mineur, "il est lourdement condamné le 18 décembre 2007 à 18 mois de prison ferme ${ }^{39}$ ». Mohamed considère que cette incarcération est une injustice et les courriers qu'il adressera à sa famille le prouvent : « Je me suis fait allumer pour dix-huit mois, injustement. C'est aussi ça qui m'a mis en colère t'as vu, quand j'ai vu l'injustice de la loi française t’as vu, 18 mois pour un sac à main alors que le proc en réclamait une année, j'en prends dix-huit et on fait tomber le sursis. Donc voilà, j’avais déjà un peu les nerfs t'as vu. Mais Hamdulillah [merci mon Dieu, gloire à Dieu], je m'en suis remis à Allah. J'ai vu que je m'étais fait balancer par une personne et si on me mettait cette affaire de X5, ça allait faire beaucoup. C'est là que j'ai invoqué Allah et, quand je suis arrivé à la gendarmerie, j’ai vu que... que les gendarmes étaient à côté de leurs pompes et j'ai vu que c'était Allah qui m'avait facilité, et c'est là le déclenchement quand je suis rentré dans l'Islam ${ }^{40}$. "

Merah précise également l’origine de sa foi : « La première fois que suis entré cela m'a apporté la foi en ALLAH et pour cette fois-là wallahi [Je te le jure] cela m'a ouvert encore plus ma foi et je regrette pas, car désormais, à ma sortie, je saurai très très précisément ce qui me reste à faire, et le hebss [prison] ça passe, je m'inkiette vraiment pas, je patiente car ALLAH aime les patients... Maintenant je vais faire ma prière tout $\operatorname{seu}^{41} \ldots »$

Les propos de Mohamed Merah sont identiques à ceux de son aîné Khaled Kelkal. En 1995, ce dernier disait déjà : "Franchement en tant qu'Arabes, la justice ne nous aime pas. Il y a une justice à deux vitesses. Le gars de Vaux-en-Vélin qui se fait attraper, il est sûr de prendre un an et demi sur les autres, ça c'est sûr. Pour moi, il n'y a pas de justice. Ce qu'ils appellent justice, c'est l'injustice ${ }^{42}$. " Nous notons chez Kelkal et Merah les mêmes types de discours et le même processus de radicalisation par la prison. De l'identité héritée (valeurs familiales que les djihadistes ont des difficultés à faire leurs) à l'identité rêvée (travail, famille, maison), et enfin à l'identité construite (délinquance, radicalisation, terrorisme), l'islam pourrait être considéré comme une opportunité de rencontre qui permet à certains jeunes de se donner « une licence » pour agir, de 
s'octroyer un pouvoir afin de transgresser tous les interdits des sociétés occidentales. Mais, cette foisci, il s'agit "d'agir au nom de Dieu », auquel il est possible qu'ils ne croient même pas. Leur adhésion à l'idéologie djihadiste serait ainsi loin de répondre "à une quête de spiritualité ». Ce qu'ils cherchent, selon Denis Jeffrey, " est moins une religion pour méditer, qu'une religion comme morale doctrinaire qui leur permet de libérer leur trop plein pulsionne ${ }^{43}$ ». Amedy Coulibaly, qui a tué une policière et quatre personnes de confession juive dans une épicerie casher à Paris le 9 janvier 2015, est décrit par ceux qui le connaissent comme quelqu'un qui était obsédé par l'argent. Un délinquant, impliqué dans plusieurs vols, qui a été condamné à six ans de prison après le braquage d'une banque. Il disait ne pas vouloir travailler sous les ordres d'un mécréant. Pour justifier ses meurtres, il a invoqué le devoir de venger le prophète ${ }^{44}$. Quant à Mehdi Nemmouche, accusé d'avoir commis la tuerie dans le Musée juif de Bruxelles le 24 mai 2014, il est décrit comme particulièrement sadique par Gilles Kepe ${ }^{45}$. Pour paraphraser Olivier Roy, on peut dire que ces jeunes "n'ouvrent pas à l'islamisation des sociétés mais à la réalisation de leur fantasme d'héroïsme malsain ${ }^{46}$ ". D'ailleurs, ils ont un " profil semblable, ils ont bu de l'alcool, fait la fête, dragué les filles, commis des actes de délinquance, ils ont connu la prison ${ }^{47}$ » et, ensuite, ils se convertissent au salafisme ou au djihadisme dans une recherche de reconnaissance sociale.

Pour David Le Breton, la morale djihadiste séduit beaucoup de jeunes parce "qu'elle propose des réponses claires et définitives pour expliquer la souffrance, le sens à la vie, la sexualité, la rédemption ${ }^{48}$ ". Les préceptes coraniques deviennent le fondement de leur émancipation, comme on peut le lire dans une lettre que Merah adresse à son frère Abdelkader : «Comme tu sais, j'ai pris 18 mois et pour te corriger, ce n'est pas une petite peine, parce que j'ai 9 mois de sursis, en plus de ça j’ai les 4 mois de mon aména-

gement, donc t'as compris en calculant avec les grâces ça me fait 16 mois plein pour un défaut de P.C. Subhanalah [gloire à Dieu]. Et si tu calcule tout ça me fait 37 mois ferme. 3 piges passées pour un gouffaparchak [un mécréant] et un défaut de PC. Subhanalâh - Subhanalâh - Subhanalâh. La hawlawa la quwwatailâhbillâh [il n'y a que la force de Dieu qui prime], mais el hemdoulillah [merci mon Dieu, gloire à Dieu], c'est une épreuve du Tout-Miséricordieux et cette injustice bi-izmillâh [au nom de Dieu] me serra bénéfique le jour dernier car iwallah [au nom de Dieu] je souhaite plus que tout qu'ALLAH me venge de ces Kofar [mécréants] !!! amine... » Au fur et à mesure de son incarcération, les courriers que Merah adresse à son frère commencent par de longues louanges à Allah : «Toutes les louanges appartiennent à ALLAH le seigneur de l'univers et la fin heureuse est certes destinée et il n'y aura de repproche que pour les... injustes. Je témoigne que nul ne mérite d'être adoré en dehors d'ALLAH l'unique, sans associer le créateur des pre-

La religion n'est qu'une rencontre entre une trajectoire accidentée en perte de vitesse et une société qui n'arrive pas à assurer à certains membres les places qu'ils espèrent avoir. La radicalisation de certains jeunes ne serait finalement que la conséquence directe de leurs situations dramatiques dans les cités.

miers et derniers et le seigneur des cieux et terre... La parole la plus véridique, le livre d'ALLAH, est la meilleure des voies à suivre, celle de Mohammad, et la pire des choses est la nouveauté. Toute nouveauté mène à l'égarement, tout égarement mène à l'enfer. ALLAH (azawajal) [Que Dieu soit exalté] dit: "Vous préferez la vie de ce monde, Alors que la vie derrière est meilleure et plus durable". Sourate 27, "Al-A'la", verset 16-1749.»

On peut voir dans ces courriers de Merah, à l'instar d'autres jeunes djihadistes, que la "référence religieuse constitue tout à la fois le fondement, le moteur et le sens de la transformation " opérée par

43. Denis Jeffrey, "La radicalisation des jeunes djihadistes ", in Denis Jeffrey et al., Jeunes et djihadisme. Les conversions interdites, Laval, Presses universitaires de Laval, 2016, p. 21. 44. David Le Breton, "Le djihadisme comme rite de virilité ", in Denis Jeffrey et al., Jeunes et djihadisme. Les conversions interdites, Laval, Presses universitaires de Laval, 2016. 45. Gilles Kepel, Antoine Jardin, Terreur dans l'Hexagone, Paris, Gallimard, 2015, p. 162. 46. Olivier Roy, La peur de l'islam, La Tour d’Aigues, éd. de L'Aube, 2015, p. 14.47. Olivier Roy, "Une révolte générationnelle et nihiliste ", in Le Monde, hors série, janvier-mars 2016. 48. David Le Breton, op. cit., p. 22. 49. Alex Jordanov, op. cit., p. 38. 
les djihadistes ${ }^{50}$. Pour eux, "il n'existe qu'une seule religion, un seul Dieu, une seule vérité. Ils n'acceptent pas la différence, la diversité, le relativisme des modes de vie $e^{51}$. "On offre aux convertis djihadistes un kit islamique simple, réinventé, réduit à quelques mots d'ordre au nom d'une guerre sainte contre les mécréants. Ce kit islamique "efface les doutes, les ambiguités, les ambivalences, et les nuances; il tranche dans le vif entre le vrai et le faux, le moral et l'immoral et il donne des réponses sans appel aux comportements requis $^{52}$ ». Il s'agirait, selon David Le Breton, d'un islam qui " potentialise la colère en lui donnant une légitimité, un cadre de pensée et d'action ${ }^{53}$ ". Toutefois, il faut faire attention parce qu'on ne peut pas interpréter les propos de Merah comme une radicalisation de l'islam, mais plutôt, comme le souligne Olivier Roy, " une islamisation de la radicalite $^{54}$ ». La religion n'est qu'une rencontre entre une trajectoire accidentée en perte de vitesse et une société qui narrive pas à assurer à certains membres les places qu'ils espèrent avoir. La radicalisation de certains jeunes ne serait finalement que la conséquence directe de leurs situations dramatiques dans les cités. Merah, Coulibaly, Nemmouche, Kouachi, etc., étaient en rupture avec leurs parents. On pourrait parler d'une révolte générationnelle contre ce que représentent leurs parents : l'échec de leur parcours en France. Or, comme ces derniers, eux aussi vivent leurs échecs comme définitifs. Le djihadisme constituerait la voie de la promotion sociale, puisque dans la communauté de daech, «ces jeunes qui n'arrivaient plus à démêler les fils de leur piètre existence, ont essayé de s'accrocher à des grappes de sens ${ }^{55}$ », et ils ont trouvé une source de reconnaissance sociale réconfortante. Enfin, il n'est pas inutile de rappeler en quoi le mirage de l'égalité des chances, conduisant à faire l'expérience du mépris, a envahi la vie de ces jeunes, «au point de les rejeter dans la résistance et l'affrontement social, autrement dans la lutte de reconnaissance ${ }^{56}$ ».

\section{Conclusion}

La violence produite par les djihadistes est un message pour l'existence sociale, une demande de reconnaissance de leur souffrance. Mais n'oublions pas, enfin, que donner un sens à son existence, ou se donner les raisons de vivre et d'espérer est un enjeu existentiel majeur. Nous pensons que les chemins de la radicalisation sont inépuisables. Entre les rencontres en prison qui tissent des liens avec des générations différentes, la volonté de Coulibaly " de se faire les policiers » qui ont tué sous ses yeux son meilleur ami Ali Rezgui en septembre2000, pendant que lui et son ami chargeaient des motos volées dans une camionnette, ajoutées à cela les explications par l'islamisation de la société, par le mirage du modèle français d'intégration, par la discrimination subie, ou par la conversion à l'islam... toutes ces explications trouvent assez rapidement leurs limites ${ }^{57}$. Nous conviendrons donc qu'il existe plusieurs facteurs entremêlés qui conduisent ces jeunes à se lancer dans le djihad et qu'au-delà des explications puisées dans les théories sociologiques (ou autres), aucune n'est généralisable à l'ensemble "car la déviance est une catégorie abstraite qui n'a pas d'unité du point de vue des comportements. Bien souvent une théorie ne vaut que pour un comportement ». Pour mieux saisir les raisons du basculement, il faut reconstituer la biographie de l'individu afin de "comprendre l'enchaînement des interactions et des événements qui l'ont marqué et l'ont prédisposé à telle ou telle transgression ${ }^{58} »$. 